Arhe XVII, 34/2020

UDK 164

DOI https://doi.org/10.19090/arhe.2020.34.85-102

Originalni naučni rad

Original Scientific Article

\author{
JOVANA KOSTIĆ ${ }^{1}$ \\ University of Belgrade, Faculty of Philosophy
}

\title{
LOGIC FOR THE THEORY OF CONCEPTS ${ }^{2}$
}

\begin{abstract}
In this paper, we follow Gödel's remarks on an envisioned theory of concepts to determine which properties should a logical basis of such a theory have. The discussion is organized around the question of suitability of the classical predicate calculus for this role. Some reasons to think that classical logic is not an appropriate basis for the theory of concepts, will be presented. We consider, based on these reasons, which alternative logical system could fare better as a logical foundation of, in Gödel's opinion, the most important theory in logic yet to be developed. This paper should, in particular, motivate the study of partial predicates in a certain system of three-valued logic, as a promising starting point for the foundation of the theory of concepts.
\end{abstract}

Keywords: intension, concept, meaningful applicability, three-valued logic, partial predicate, connective

\section{INTRODUCTION}

The theory of concepts, or concept theory, stands in this paper for a theory envisioned by Kurt Gödel that deals with the formal properties of concepts and with the relation of concept application (Gödel, 1944; Wang, $1996^{3}$, ch. 8). Under concepts, the properties and the relations

\footnotetext{
${ }^{1}$ Author's e-mail address: kostic.jovana345@gmail.com

${ }^{2}$ Work on this paper was supported by the Ministry of Education, Science and Technological Development of the Republic of Serbia through the project Dynamic Systems in Nature and Society: Philosophical and Empirical Aspects (No. 179041)

${ }^{3}$ This book contains Hao Wang's records of the views Gödel held during their conversations. Gödel's remarks documented in this book will be referred to by
} 
between entities are understood that form the intensional meaning of predicates. Gödel regarded concepts as the entities on a par with sets, which are independent of our mental activities and of the language in which they can be characterized (Gödel, 1944, p. 128). They should, like the sets, have some objective, formal properties that can be studied in a logical theory. These properties of concepts are, presumably, grounded in their intensional nature. What is the nature of concepts, and which formal properties do they have, is something we have an intuitive understanding of, but do not yet fully grasp. By developing this rudimentary understanding of concepts and making it more precise, the theory of concepts is supposed to shed light on these matters. Gödel has made quite a few remarks on this theory, mostly emphasizing the importance of establishing it and the special place among the logical theories it should eventually acquire. But he has also made a few notes on how he imagined such a theory could look like and on the questions it should deal with. Following these notes, we try to distinguish some key features of a logical basis for such a theory, and consider which logical system is best suited to this role.

What crucially distinguishes the theory of concepts from other mathematical and logical theories, is that it deals with the fundamental intensional entities and relations, that is, with the notion of intension itself. Gödel imagined the theory of concepts as an intensional analogue of set theory, a theory dealing with the thoroughly extensional entities sets and with the relation of set membership. Sets are taken to be the collections of entities that lack any internal structure. The entities belonging to the same set can share some properties, or stand in particular relations to one another, in other words, they can fall under the same concepts. However, their membership in a set should not be taken to depend on these properties and relations, or to indicate them. According to the Zermelo-Fraenkel set theory, sets are completely determined by their members. They are made bottom-up, by gathering some previously existing, simpler, entities and making a unity out of them, and not by specifying some property and picking out, from the entire universe of

the numbers they are given there. 
entities, those that have this property (even though the properties of its members can be used to characterize an already formed set and pick it out from the universe of sets). The properties and the relations between the elements of a set are not the subject matter of set theory, but should rather be studied in the theory of concepts. The relation of falling under a concept (i.e., of concept application), which should present a formal rendition of the phenomena of having some property and standing in a particular relation, can be seen as an intensional counterpart of the relation of set membership. The differences in the nature of these two relations, that are supposed to show in their formal properties, should be brought to light by the theory of concepts.

Gödel suggests that in establishing the theory of concepts, the example of set theory is to be followed (Wang, 1996, 8.6.18). Set theory is built as an extension of the classical predicate calculus with the relation of set membership and the axioms describing it, from which the formal properties of sets can be inferred. The theory of concepts could, in Gödel's opinion, be founded in a similar way, if instead of the relation of set membership, the relation of concept application is added to the underlying predicate calculus and described by some new axioms. He thought that the search for the axioms describing the relation of concept application is what the search for the theory of concepts consists in, since to understand what the concepts are is to understand what an application of a concept to an entity involves, and how it differs from the membership of this entity in a set (cf. Crocco \& Bernard, 2016, p. 154). Gödel seems to have been confident that the classical predicate calculus is equally suitable as a logical basis for the theory of concepts. There may be different reasons in favor of this view, but Gödel's main reason most probably was that there is no alternative logical system that could compare in strength to the classical predicate calculus. Any other logical system seems to make some important mathematical principles and modes of reasoning unavailable in a theory built on it. Given the Gödel's view that the theory of concepts is to become the central theory in logic, and that it might even contain set theory (at least if it turns out that every set is the extension of some concept), his reluctance towards the change of logic seems reasonable. However, if we set aside the discussion of the 
potential role and status of the theory of concepts among other logical or mathematical theories, and focus only on its subject matter, then we can find several reasons to question the adequacy of the classical logic as its logical foundation.

In what follows, we consider some important logic-related questions and topics which should be dealt with in the theory of concepts, and which might suggest the properties that an appropriate logical foundation of this theory should have. Some reasons for understanding logical words in a more intensional way, will be indicated throughout, and a possible explanation of what this more intensional way of understanding is, will be proposed. The logical systems that seem best to conform to the derived requirements, are considered in parallel.

\section{THE THEORY OF CONCEPTS AS AN INTENSIONAL THEORY}

The most general reason for believing that the theory of concepts is in need of some nonclassical logical foundation is that it is supposed to be an intensional theory, a theory dealing with the notion of intension itself. Classical logic is, on the other hand, thoroughly extensional. It disregards any aspect of the meaning of its expressions other than their extension. Formulas (i.e., sentences) of classical logic are thus interpreted as being either true or false, and logical connectives are understood as the truth functions, that is, as the functions that map the set of the two truth values to itself. The meanings of predicates are identified with the sets of entities to which they can be truthfully ascribed, and predication is taken to express the membership in a set corresponding to the predicate in question.

As thoroughly extensional, classical logic forms an appropriate basis for mathematical theories that deal with extensional objects, such as sets. On the other hand, since the theory of concepts deals with the objects that are supposed to provide the expressions with their intensional meaning, it seems reasonable to inquire whether it necessitates some logical foundation that does not neglect this aspect of meaning of its expressions. But which other, more intensional, feature of formulas and other expressions should be taken into account in a logic that is supposed 
to form a more appropriate basis of an intensional theory? Which of these features can influence the logically relevant behaviour of formulas?

Classical logic deals only with the formulas that have an extensional meaning, that is, that are either true or false. However, if we are interested in the intensional meaning of formulas and their constituents, then we might also want to consider the formulas that, in virtue of that meaning, lack a truth value. Logic that answers this requirement could be some three-valued logic in which the third value, besides truth and falsity, is interpreted as the lack of classical truth value. The intensional meaning of formulas, and the expressions constituting them, would be acknowledged in a theory built on this logic by its acceptance and a study of the formulas whose lack of the extensional meaning can only be explained by the intensional meaning of the formulas and their constituents. This study could reveal some properties of the intensional meaning of expressions and its relation to their extensional meaning as, for example, what does it take, in terms of the intensional meaning of its constituents, for a formula to have a truth value. How is all this related to the study of concepts and the relation of concept application, is explained in the next section.

\section{Predicates interpreted as concepts}

The simplest, atomic, formulas of predicate logic are made by the application of a predicate constant to one or several names. There are no restrictions in the formation of these formulas, except that the number of names to which a predicate is applied has to correspond to its arity. If every formula built in this way should receive a truth value, then every predicate has to be taken to be either correctly or incorrectly ascribable to an adequate number of names, yielding a true, or a false sentence, respectively. While this fits well with some interpretations of predicates and predication, others seem to require some additional possibilities.

There are two ways in which predicates can be understood either extensionally, as standing for sets, or intensionally, as expressing the concepts. The predication is accordingly interpreted either by the set membership relation, so that it is used to express that some entity, or 
several of them, belongs to a particular set, or by the relation of concept application, so that it ascribes a particular property or a relation to some entities. It seems safe to suppose that every entity either does, or does not, belong to a particular set. So, if the predication is understood as the statement about the membership in a set, then every sentence it results in should be regarded as true or false. The situation is not that clear when it comes to the intensional interpretation of predication. Stating that some entity falls under a concept or denying that it does, seems to have more significant implications for this entity. In particular, both options seem to imply that the concept in question can be meaningfully (although not necessarily truthfully) applied to this entity, that is, that the statement of this application yields a meaningful proposition. However, there may be some cases in which this does not hold. Consider, for example, the statement that a particular building is numerous. Understanding and evaluating the meaning of this statement according to the extensional interpretation of predication, seems to be unproblematic - it expresses a false proposition about the membership of this building in a set picked out by the specification of the property its members have. On the other hand, if this statement is interpreted as an attribution of some properties to an entity, in virtue of which it can be described as numerous, then it seems more difficult to ascribe any coherent meaning to it, that is, to understand a proposition expressed and the conditions for it being true. There seems to be something wrong with this statement besides it not being true, since saying that a building is numerous and saying that it is not numerous, if interpreted intensionally, seem to be defective in the same way. It is not only that a concept of being numerous happens not to apply to a building, but it might not even be meaningfully applicable to it. If this is the case, then we should consider both the sentence expressing this application and its negation as being neither true nor false, but meaningless. This might hold as well for the statement that a set is red, or that an average taxpayer lives in New York, that a concept of a horse is alive, and other so-called category mistakes. ${ }^{4}$

4 Incidentally, these possibly meaningless applications of concepts bear similarities to the applications of the algorithm to the entities for which they are 
Whether or not the given examples really contain meaningless applications of concepts, they do seem to suggest that much more is implied by a statement that some entity falls under a concept, than by a statement that it belongs to a set. It is, hence, possible that there are some conditions which the statements of the first kind have to satisfy to be meaningful, that do not apply to those of the second kind. This may provide a part of an explanation of the way in which the relation of set membership and the extensional interpretation of predication differ from the relation of concept application and the intensional interpretation of predication: stating that something is or is not an element of a set is either true or false, while stating that it falls under a concept can be neither true nor false, but meaningless. Interpreting a predication as a statement that an entity falls under a particular concept thus means allowing for this predication to be meaningless. An intensional interpretation of predication would then imply that the predicates in question are understood as partial predicates, that is, as the predicates that can yield a truth-valueless sentence when applied to particular names.

The idea that the meaningful applicability of concepts could be problematized is mentioned in Gödel's comments on the possible solution to the intensional paradoxes (Gödel, 1944, p. 137). It accords well with his view on concepts according to which, contrary to sets, concepts do not presuppose for their existence the entities to which they apply, and the results of these applications. They can, as such, exist and be wellunderstood even if the question to which entities they (meaningfully) apply is still open.

One important theory that deals with the meaningful applicability of concepts, is the theory of types. The concepts are in this theory divided into disjoint types according to the entities they are meaningfully applicable to, so that a concept belonging to a particular type can be meaningfully applied only to the concepts or entities of some lower type. This theory is too restrictive according to Gödel, since it takes concepts to

not defined, which result in non-terminating procedures. The study of such procedures can be revealing in regard to the algorithms and the way they differ from functions, which can always be made total by an arbitrary ascription of the values to the arguments for which the corresponding algorithm is undefined. 
be meaningfully applicable only to the entities belonging to some predetermined class of entities and precludes any self-applicability of concepts (Gödel, 1944, p. 137). The theory that would take the abovementioned sentences to be meaningless, would maybe put even more stricter restrictions on the meaningful applicability (since it would suggest dividing the entities into distinct categories and limiting the meaningful applicability of concepts to the entities from a particular category). As such it might, in Gödel's eyes, fail to be a promising candidate for the theory of concepts. Gödel thought that instead of overly restricting their meaningful applicability, we can take concepts to be meaningfully applicable "everywhere except for certain 'singular points' or 'limiting points'..." (Gödel, 1944, p. 138). How should we judge if some application is a limiting case, is not that clear. One option is to take into account the consequences of these applications, and pronounce meaningless those whose consequences are unacceptable. For example, some instances of these limiting points of meaningful applications can be taken to lead to the intensional paradoxes. The unacceptable consequences of the applications in this case would be the outright inconsistencies.

Self-applicability of concepts and intensional paradoxes

A characteristic of the applications that are in the basis of intensional paradoxes is that they are self-referential, that is, those are the applications of particular concepts to themselves. The paradox to which Gödel pays most attention is the paradox of the concept of concepts not applying to themselves, which is an intensional variant of the Russell's paradox of the set of sets that are not members of themselves. If the concept of concepts not applying to themselves applies to itself, then it has the property contained in this concept, and this is to not apply to itself. On the other hand, if it does not apply to itself, then, in virtue of this property, it does apply to itself. Either way we end up in a contradiction. The assumption of the self-reference in the basis of intensional paradoxes makes them similar to the extensional paradoxes pertaining to sets that are based on the assumption that there are sets that 
can be members of themselves. According to the accepted set theory, no such set actually exists. This is explained by the fact that sets are inductively built from some previously existing entities that in turn become their elements. No set built in this way can contain itself, since it does not exist prior to its own formation. So, the extensional paradoxes are resolved in set theory which has shown that they are the consequences of the wrong assumptions about the nature of sets. When it comes to the intensional paradoxes, the solution that would consist in denying the possibility of a meaningful self-referential application, seems to be unmotivated (Wang, 1996, 8.6.20; 8.6.21). As Wang reports, Gödel has thought that there is nothing in the nature of concepts that would make impossible for them to apply to themselves. Concepts are not inductively built from the entities to which they apply, and their meaning does not depend on these entities. The possibility of self-application can thus be a property by which the nonextensional and noninductive nature of concepts comes to light, and which, as such, should not be neglected in the theory that attempts to elucidate the nature of concepts. Besides that, there are also numerous examples of concepts that seem to apply to themselves: "the concept of concept, the concept of being applicable to only one thing (or one object), the concept of being distinct from the set of all finite mathematical sets, the concept of being a concept with an infinite range, and so on" (Wang, 1996, 8.6.3). Taking all this into account, it seems that the only way to answer Gödel's request that the solution to intensional paradoxes reflects the nature of concepts, is to find a solution that allows self-applicability of concepts, but restricts it in some other way so that the intensional paradoxes are avoided.

Instead of showing that no concept can be meaningfully applied to itself, the contradictions to which the self-referential applications in the basis of paradoxes lead, can be taken to show meaninglessness only of these particular applications. The idea that the paradoxes are the consequence of regarding meaningless sentences as true or false, is wellknown (see, for example, (Bochvar, 1981) for such a solution to the extensional and semantic paradoxes). However, this solution seems to be particularly well suited to the intensional paradoxes, since they are most directly concerned with the meaning that some expressions may lack. The 
study of meaningful applicability could thus, by proposing the solution to these paradoxes and with it overcoming the major difficulty in the development of the theory of concepts, potentially set the foundation for the future theory, which is something Gödel seems to have expected from an appropriate solution to the intensional paradoxes (Wang, 1996, p. 273).

The problem of meaningful applicability could be dealt with inside a classical theory (see: Reinhardt, 1986; Feferman, 1984). However, its study in a three-valued logic with the partial predicates could make a more appropriate starting point for the foundation of the theory of concepts, since a theory whose predicates are partial can be taken to reflect the properties of concepts and their possibly meaningless applications. Besides that, there are some other questions, to which we now turn, to be dealt with in the theory of concepts, that could also make a three-valued logic more appropriate as its logical foundation.

\section{The intensional meaning of connectives}

The lack of truth value is not a distinctive mark of the meaningless sentences. Sentences of some other kinds, we would regard as meaningful, also seem to be without classical truth value, such as the sentences ascribing vague concepts to some entities, the sentences referring to future events, and similar. The meaninglessness of the sentences should, in addition to their lack of truth value, also be shown in the effect it has on the complex sentences made from them by the connectives. A three-valued logic proposes a nonclassical interpretation of the logical connectives that takes into account the possible lack of meaning of the sentences they apply to. Owing to its interpretation of connectives, this logic might come to form a basis for a theory of concepts that could assumably better accommodate additional requirements for such a theory, such as giving an account of the way the complex concepts are formed, and describing the relations between them. 
Formation of concepts

The characteristic of concepts, that they can be combined so as to form some complex entity - another concept or a proposition, is according to Gödel, essential for their understanding (Crocco et al., 2017, p. 1). So, in addition to describing the application of concepts, and the conditions under which it is meaningful and thus results in a proposition, another central task for the theory of concepts would be to describe the way complex concepts are built from some simpler ones. This task would resemble the one pertaining to sets, which is accomplished in set theory with its description of the way some sets are formed from others by the application of different operations. Logical connectives have an important role in this description. An intersection is described with the use of conjunction: something belongs to the intersection of two sets if and only if it belongs to both of them; a union of sets is similarly described with the use of disjunction; and a complement of a set with the use of negation. The logical relations between these connectives determine the relations between the sets made by these operations. Namely, in the basis of some set identities lie the logical truths in the form of equivalences containing the mentioned connectives.

Logical connectives seem to have an important role in the formation of complex concepts as well: "A concept is a whole composed of primitive concepts such as negation, conjunction, existence, universality, object, the concept of concept, the relation of something falling under some concept (or of some concept applying to something), and so on" (Wang, 1996, 8.6.17). The role of the connectives in the formation of concepts seems to be even more pronounced - they have an important role to provide a concept with the structure (cf. Crocco et al., 2017 , p. 1, fn). It is thus to be expected that the logical connectives figure prominently in the description of the concept formation. It seems easy to find the examples of the concepts in whose formation connectives clearly participate. For instance, the concept of being non-physical seems to be composed by negation from the concept of being physical; the concept of being a flightless bird seems to be composed by conjunction and negation from the concepts of being a bird and having the ability to fly; the 
concept of having a (classical) truth value is arguably composed by disjunction from the concepts of being true and that of being false, etc. Should these connectives that have a role in the formation of concepts be understood in the same way as in set theory? Or should their meaning differ in a way that makes them more suitable to be used in the formation of concepts, instead of sets?

It is clear that a concept made by conjunction from two other concepts should apply to the entities to which both of these concepts apply and fail to apply to those to which any of them does not apply. Similarly, a concept made by negation from another concept should apply to the entities to which this concept does not apply, and fail to apply to those to which it applies. But, if we accept that some applications of concepts are meaningless, then we also have to consider which consequences this has for the applicability of concepts made from them. This can be where the difference in the role connectives have in the formation of concepts and in the formation of sets, appears. To understand the process of forming the concepts by connectives, we would have to consider how do these connectives behave when applied to the sentences expressing the meaningless applications of concepts.

There is a strong intuition that a complex sentence made from some meaningless one should be meaningless as well. An interpretation of the connectives that conforms to this intuition is given by the Kleene's weak three-valued logic (Kleene, 1971, p. 329 and p. 334). The formulas of this system can be either true, false, or they can be without a truth value. Those formulas that lack a truth value can be interpreted as meaningless or nonsense (see Bochvar, 1981). The connectives of the system, when restricted to the formulas that are true or false, resemble the classical connectives. But, in their new interpretation, the classical truth tables are extended to include the cases when the connectives apply to the formulas without a truth value, and in each such case the resulting formula is taken to lack a truth value as well. This interpretation of the connectives seems to accord well with the role they should have in the formation of concepts. A complex concept made from some simpler ones should supposedly have some coherent meaning constructed from the meanings of these concepts. If any of these concepts fails to be 
meaningfully applicable to an entity, then it is not clear which meaning could be given to the ascription of a complex concept made from it to the same entity. For example, which complex property can be taken to be ascribed to the number five by the sentence The number five is either blue or prime, or to the sets by the sentence All sets are smelly?

One additional problem concerning the formation of complex concepts, that apparently cannot be tackled or solved by the introduction of a three-valued logic, is still worth mentioning. It seems that not every combination of concepts meaningfully applicable to an entity results in a concept that is meaningfully applicable to the same entity. Take the sentence The room is spacious and not spacious, for an example. Which property is ascribed to the room in this sentence? Is it some contradictory property? Should the concepts including these properties be taken to exist at all? If we are not willing to accept such concepts, and the possibility of their meaningful application to some entities, then we can take this example to point out the need for restricting the application of the general principles for the formation of complex concepts by the connectives. As Gödel remarked: "What is wrong is not the particular ways of formation, but the idea that we can form concepts arbitrarily by correct principles" (Wang, 1996, 8.5.20). It is, in Gödel's opinion, an arbitrary formation of concepts that has led us to the intensional paradoxes. If this is so, then what should lead to their solution is an introduction of the correct principles restricting the formation of concepts.

In addition to the connectives mentioned in this section negation, conjunction and disjunction, there are other important logical connectives - implication and equivalence. These connectives might have a role in a description of the relations between the concepts, rather than in their formation. Which meaning should these connectives assume to fit this role?

Relations between concepts

Aside from the principles according to which complex concepts are formed and their restrictions, the theory of concepts should also deal with the relations between concepts. Some relations in which concepts 
stand are assumably grounded in their meaning, and could be crucial for its understanding. This external perspective could actually turn out to be indispensable when the meaning of concepts is in question, since, given that concepts are not inductively built, their meaning does not depend on the entities to which they apply, but is rather determined by the relations in which they stand to other concepts. Such are, for example, the relations between a complex concept and the concepts from which it is built. These relations can supposedly be expressed by the implications and equivalences, stating what follows from the fact that some concept applies, or is meaningfully applicable to an entity, about the concepts that participate in its formation, or that are in some other way related to it. For these relations to be expressible with the use of implication and equivalence, these connectives would have to take on some nonclassical meaning.

In classical logic, an implication is true if and only if its antecedent is false, or its consequent is true. So, its truth value does not depend on the intensional meaning of its antecedent and consequent, and the existence of any conceptual connection between them. The same is the case with classical equivalences - they are true if and only if their two subsentences have the same truth value. Such sentences cannot be taken to express the connections and the relations grounded in the meaning of concepts. For example, even though the sentence If something is a unicorn, then it is a set is true when implication is classically understood (since its antecedent is false for every entity), we would not want to admit that there is some conceptual connection between the two sentences, even less that it describes some connection in meaning between the concept of unicorn and the concept of set. If we took classical implications and equivalences to describe the connections between concepts, then we would have to accept that there are some necessary connections between concepts stated by the classical logical truths concerning the two connectives. For example, the reasoning that establishes the truth of the above sentence can be formalized by a logical truth of the form . Logical truths would also be all the sentences of the form $B \rightarrow(A \rightarrow B)$, $(A \rightarrow B) \vee(B \rightarrow A)$, etc., which, if taken to express the connections between the concepts whose application to an arbitrary entity is expressed 
by $A$ and $B$, would all have some unacceptable consequences. Understanding equivalence as a function of the two truth values leads to similar difficulties - meanings of too many concepts would have to be taken to be related, as, for example, the fact that any sentence of the form $(A \leftrightarrow B) \vee(A \leftrightarrow \neg B)$ is a logical truth, implies. To avoid these unwelcome consequences, in describing the connections between concepts, we should rather use some connectives whose interpretation takes into account the intensional meaning of the sentences they connect.

In Kleene's weak three-valued logic, an interpretation of these connectives is proposed that seems to be at least a step in the right direction, since it takes into account the possible lack of meaning of the sentences to which these connectives apply. For an implication to be true, it is not enough that its antecedent is false, or that its consequent is true. Besides that, the other constituent of the implication has to be meaningful as well. Some of the problems mentioned in the previous paragraph would be escaped, if the connectives are understood in this way. Namely, no classical logical truth would be a logical truth in this interpretation (since every such formula would be meaningless whenever some of its subformulas is meaningless), so the so-called paradoxes of material implication, and similar unwanted consequences of the classical interpretation of equivalence, would be avoided.

On the other hand, it is not clear that Kleene's weak implication and equivalence would be the most suitable for expressing the connections between concepts. Given that we might like to state a connection in meaning of some concepts that are not everywhere meaningfully applicable, and that it is plausible that these concepts are meaningless for the same entities, we might want that some implications and equivalences containing the sentences expressing the meaningless applications of these concepts, turn out true. Such implication and equivalence would have to differ in this from their weak versions.

To sum up, the interpretation that the connectives receive in classical logic does not seem to fit the role they are supposed to have in the theory of concepts. For that, they would have to be understood in a more intensional way, that is, as the connectives that somehow relate the meanings of the sentences to which they apply. It is assumed that some 
kind of a three-valued logic can be more successful than classical logic in answering this request, since it recognizes the possible lack of meaning of the sentences to which the connectives apply, and its consequences. Due to this, it could make a basis for a study of the consequences which the meaningless application of concepts has for other concepts and their mutual relations - the study that could be very significant for the future theory of concepts.

\section{CONCLUSION}

The discussion in this paper seems to suggest that an appropriate logical basis for the theory of concept, given its subject matter and the problems it should deal with, might be a three-valued logic in which the third, nonclassical, value is assumed by the meaningless formulas. An example of such a system is Kleene's weak three-valued logic. The predicates of this logic would be partial, which means that their application can result in a meaningless sentence. If it is assumed that an important feature of the concepts is that they are not meaningfully applicable to an arbitrary entity, then their representation by the partial predicates seems to be the most appropriate. The relation between the partial predicates and the concepts could be expressible in the theory of concepts by ax axiom saying that a concept $C$ is meaningfully applicable to some $x$ if and only if a partial predicate expressing this concept yields a meaningful sentence when applied to the name of $x$; and the one saying that the concept $C$ applies to $x$ if and only if the sentence resulting from the ascription of the corresponding predicate to the name of $x$, is true. A variant of comprehension principle might also hold in this theory stating, for every predicate, that there is a concept applicable to exactly those entities to whose names this predicate is truthfully ascribable (cf. Reinhardt, 1986, p. 223). A theory with partial predicates could thus create the foundation for the study of meaningful applicability of concepts, in which Gödel saw the possibility of resolving the intensional paradoxes and thus overcoming the main difficulty in the development of the theory of concepts. The theory of concepts could in this way be achievable as a consistent type-free theory, in which the meaningful self- 
referential application of concepts is allowed.

An attempt to found the theory of concepts, which might have such an importance as Gödel thought it would, on a nonclassical logical basis, might seem unfortunate from the start. Even if this is the case, the previous discussion could turn out to be helpful in pointing out the ways in which the classical predicate calculus could be altered, so as to resemble some aspects of a three-valued logic with partial predicates, that make it a more suitable basis for the study of the important questions concerning the nature of concepts.

\section{REFERENCES}

Bochvar, D. A. \& Bergmann, M., (1981) “On a Three-Valued Logical Calculus and its Application to the Analysis of the Paradoxes of the Classical Extended Functional Calculus", History and Philosophy of Logic Vol. 2, No. 1-2, pp. 87-112.

Crocco, G. \& Bernard, J., (2016) "Gödel and the Paradox in Max Phil X" in Kurt Gödel: Philosopher-scientist, 978-2-85399-976-2, hal-01473451.

Feferman, S., (1984) "Toward Useful Type-Free Theories I", The Journal of Symbolic Logic Vol. 49, No. 1, pp. 75-111.

Grocco, G., van Atten, M., Cantu, P., Engelen, E. M., (2017) "Kurt Gödel Maxims and Philosophical Remarks Volume X", hal-01459188.

Gödel, K., (1944) "Russell's Mathematical Logic", in: Collected Works, II: Publications 1938-1974, Oxford University Press, Oxford (1990), pp. 119-141.

Kleene, S. C., (1971) Introduction to Metamathematics, Wolters-Noordhoff, Groningen.

Reinhardt, W. N., (1986) "Some Remarks on Extending and Interpreting Theories with a Partial Predicate for Truth", Journal of Philosophical Logic Vol. 15, pp. 219-251.

Wang, H., (1996) A Logical Journey: From Gödel to Philosophy, MIT Press, Cambridge, MA. 


\section{JOVANA KOSTIĆ \\ Univerzitet u Beogradu, Filozofski fakultet}

\section{LOGIKA ZA TEORIJU POJMOVA}

Sažetak: U ovom radu sledimo Gedelove napomene povodom predviđane teorije pojmova kako bismo odredili koja svojstva bi trebalo da ima logička osnova takve teorije. Diskusija se formira u krugu pitanja o prikladnosti klasičnog predikatskog računa za tu ulogu. Biće prikazani neki razlozi koji navode na mišljenje da klasična logika nije prikladna osnova za teoriju pojmova. Na osnovu tih razloga, razmatramo koji bi alternativni logički sistem bio pogodniji za logičko zasnivanje onoga što bi, po Gedelovom mišljenju, bila najznačajnija teorija u logici koju bi tek trebalo razviti. Ovaj rad za cilj bi naročito imao da podstakne proučavanje parcijalnih predikata $\mathrm{u}$ jednom sistemu trovalentne logike, kao obećavajuće početne tačke za zasnivanje teorije pojmova.

Ključne reči: intenzija, pojam, smisaona primenljivost, trovalentna logika, parcijalni predikat, veznik

Primljeno: 1.9.2020.

Prihvaćeno: 11.11.2020. 